\title{
Les politiques du corps: I'approche critique de Didier Fassin à l'épidemie du Sida en Afrique du Sud
}

\author{
The politics of the body: Didier Fassin's critical approach to \\ the AIDS epidemic in South Africa
}

\author{
Sara Leon Spesny \\ Doctorante, Programme Phoenix JDP Dynamics of Health and Welfare/ \\ École des Hautes Études en Sciences Sociales. \\ sara.leonspesny@ehess.fr
}

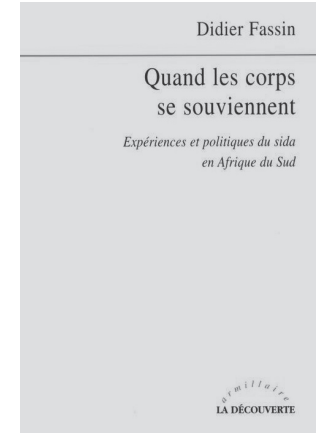

FASSIN, Didier. Quand les corps se souviennent: expériences et politiques du sida en Afrique du Sud. Paris: La Découverte. 2006. 448p.

\section{$\mathrm{L}$} 'étude de la santé comme enjeu politique et le parcours de l'histoire du sida en Afrique du Sud dans le contexte post-apartheid est l'objet de cet ouvrage. L'auteur présente l'expérience de personnes atteintes du sida qui racontent comment ils vivent leur maladie. Il met aussi en évidence les inégalités sociales, raciales et de genre dans l'Afrique du Sud contemporaine.

Fassin nous montre comment la réalité du sida en Afrique du Sud ne peut être appréhendée qu'à partir d'une ethnographie informée par une historiographie. Son but est de souligner la pluralité des points de vue, les tensions et contradictions des expériences locales ainsi que les différents acteurs, dans un double souci de rendre compte de la diversité des problématiques et de la généralité des enjeux.

L'auteur s'efforce de démontrer que la maladie n'est pas, en soi, une fatalité biologique mais l'expression même de ces inégalités qui réduisent le champ des potentialités pour les populations noires sud-africaines. "Ces inégalités devant la mort, sont d'abord des inégalités devant la vie, et mettent en évidence un état de fait inégal et violent qui affecte les corps et qui met à l'épreuve les plus faibles" (p.396). En effet, le cas du sida en Afrique du Sud met en évidence l'existence d'une politique spécifique, dite "nécropolitique", 1 pour gloser sur la formule foucaldienne de "biopolitique".

Cette situation se trouve au cour de toute politique publique: la vie et la mort des citoyens passent par l'estimation du nombre des morts et des vivants; elle comptabilise les victimes légitimes.

Le taux de contagion du sida a scandaleusement augmenté en une décennie. Entre 1990 et 2000 les statistiques ont évolué, passant de "données rassurantes" à des données catastrophiques. Néanmoins, ces statistiques nous montrent comment le sida frappe la société africaine de manière inégale. Les risques d'être contaminé et la durée de survie sont étroitement liés aux conditions de vie et à l'environnement matériel et humain. Par conséquent, agir sur l'environnement, les conditions de vie et la distribution de ressources se révèle tout aussi important que d'administrer des antirétroviraux. 
Dans l'Afrique du Sud post-apartheid, alors que les libertés s'étendent, les inégalités sociales s'accroissent, et la violence, même si elle se manifeste différemment, persiste dans le quotidien des populations noires sud-africaines. Cette période se caractérise donc par une tension entre une idéologie animée par la logique du racisme et un pragmatisme fondé sur la logique capitaliste.

En 1996, un débat radical, hétérogène et polémique se greffe sur l'histoire politique du sida en Afrique du Sud. L'apparente confrontation entre les "orthodoxes" et les "hétérodoxes" est analysée de près pour dégager des traits distinctifs au niveau des logiques, des savoirs et des implications politiques. Néanmoins, les "orthodoxes", désignant la communauté scientifique internationale garante du savoir et de la rationalité, défendent une vérité scientifique dont la contestation relève selon eux d'une "irrationalité pathétique". La contestation incarnée par les "hétérodoxes" est représentée par une minorité de scientifiques dont la position a été reprise par les autorités sud-africaines et plus spécifiquement par le président Thabo Mbeki ainsi que d'autres hauts fonctionnaires qui ont notamment adopté ce discours de "la pauvreté comme source de la maladie".

Les positions des "hétérodoxes" et de l'opinion publique sud-africaine ont été fortement marquées par un scepticisme envers les autorités médicales internationales. Sur ce point, l'auteur expose comment plusieurs essais cliniques financés par des organisations internationales et des pays développés ont ignoré les critères éthiques, dévoilant ainsi "une certaine continuité de pratiques racialement différenciées, voire ouvertement racistes, dans le monde médical" (p.151).

En outre, l'auteur réalise une approche historique du traitement des corps noirs pour montrer comment la santé publique s'est mise au service d'une politique ségrégationniste, teintée de préjugés moraux "blancs". En effet, la médecine a collaboré avec l'ancien régime, de manière active et passive. Cet historique des épidémies en Afrique du Sud retrace aussi l'histoire de la sanitarisation de la ségrégation.

Par ailleurs, l'auteur s'engage dans une critique approfondie du culturalisme, car la logique culturaliste présente dans les domaines scientifiques et politiques peut effectivement viser à justifier ces politiques ségrégationnistes et discriminatoires.

Ainsi, pour le monde blanc, le corps africain est toujours facteur de risque; c'est par sa force de travail qu'il s'intègre à la société contemporaine selon la dialectique racisme-capitalisme.

En effet, comme le titre l'indique, la problématique du corps représente le premier angle de l'ouvrage. D'ailleurs, c'est le corps et, notamment, l'histoire inscrite dans les corps qui font ici objet d'analyse. Ce sont les corps qui subissent la maladie, ce sont aussi les corps noirs qui portent l'histoire du racisme et des politiques ségrégationnistes. À ce sujet, Fassin constate la centralité des corps et de la mémoire: "Des inscriptions du temps dans la corporéité des individus, dans les déterminations sociales de leur destin biologique, dans les remémorations par lesquelles ils cherchent à donner un sens à leur présent" (p.14). Le souvenir, angle secondaire de l'intitulé, renvoie à l'évidence incontournable du poids du passé qui dépasse le temps et investit le présent. C'est alors que le passé se dévoile et que la trace du passé résiste à l'oubli. C'est ainsi que "les corps se souviennent".

Face à l'interprétation de ce "souvenir" des corps, l'auteur propose une théorie de "l'incorporation du monde social", qui vise à démontrer la manière dont "l'histoire s'inscrit 
de la façon la plus subreptice et la plus décisive dans les interstices de la vie quotidienne, dans les discours et les actes, dans les représentations et les pratiques" (p.332). En référence à la sociologie de Pierre Bourdieu, de l'anthropologie de Thomas Csordas et de la "phénoménologie de la perception" de Maurice Merleau-Ponty, on peut retrouver une articulation entre l'expérience, en tant qu'inscription corporelle dans le monde, à la fois individuelle et collective, et les conditions de vie inscrites dans une réalité économique et sociale.

Ici la mémoire se nourrit de l'histoire et vice-versa: "les politiques du passé comme étant à la fois la source et le produit de cette activité collective qui consiste, d'une part, à délimiter l'histoire et la mémoire, et, d'autre part, à construire les relation entre elles" (p.195). D'ailleurs, c'est à travers l'interprétation psychanalytique du "retour du refoulé" ou "le mort hante le vif", que le passé est toujours à l'œuvre dans le présent. C'est donc sur un double processus contradictoire que la mémoire se fonde: l'oubli (compris comme une action contre le passé) et la trace mnésique (le retour de cet oubli).

L'histoire sud-africaine oscille entre oubli et mémoire, passé et présent, vie et mort. Les efforts pour reconstruire un présent impliquent d'imposer l'oubli à un passé trop douloureux. Cependant, la logique de l'apartheid est autrement plus difficile à effacer et l'épidémie du sida le montre bien.

Dans sa particularité historique, le cas du sida en Afrique du Sud forme une sorte de prisme qui fait apparaître la logique des inégalités et la légitimation de ces injustices sociales déterminée par des territoires, régions et pays. Ainsi une frontière morale définit les politiques de violence, de souffrance et d'exclusion sociale.

Didier Fassin s'attache à dépeindre le contexte historique et politique du sida en Afrique du Sud. Sa contribution sans doute la plus significative repose sur une interprétation à la fois politique et phénoménologique, portée par des personnages invisibles politiquement et insignifiants socialement qui, en racontant leur propre histoire, donnent une voix au sida.

L'intention de l'auteur est essentiellement de présenter le travail anthropologique comme une discipline qui requiert un engagement moral fort; l'anthropologue devient un acteur politique, scientifique et moral. C'est grâce à son regard politique et moral que l'anthropologie acquiert son utilité sociale. Ainsi, l'auteur signifie son ambition : la discipline anthropologique doit viser justement à signaler les injustices du monde, à confronter les inégalités avec la parole scientifique et à lutter contre "l'anesthésie politique". Quelle est la portée de ce travail anthropologique, dénonciateur mais intellectuel et élitiste? Ce point reste à discuter.

Il est vrai - comme le remarquait Norbert Elias - que l'anthropologue doit chercher une position entre "l'engagement" pour une cause moralement défendable et la "distanciation" qu'implique son travail scientifique.

En conclusion, la conjonction entre histoire et anthropologie permet une analyse pertinente du contexte politique et social. C'est l'une des conditions qui permet au travail anthropologique de se diffuser et de demeurer durablement dans la réflexion. Le recours à l'interdisciplinarité réussit à mettre au premier plan le sida dans son contexte politique sud-africain en même temps que dans la mémoire, le souvenir, les corps et les expériences des anonymes du monde.

\section{NOTE}

${ }^{1}$ Concept repris par Didier Fassin et développé par Achille Mbembe. 